ÉGYPTE monde arabe

\section{Égypte/Monde arabe}

30-31 | 1997

Les visions de l'Occident dans le monde arabe

\title{
Les droits de l'homme en Égypte
}

Dynamiques de relocalisation d'une référence occidentale

\section{Dina El-Khawaga}

\section{(2) OpenEdition}

\section{Journals}

Édition électronique

URL : https://journals.openedition.org/ema/1649

DOI : $10.4000 /$ ema. 1649

ISSN : 2090-7273

Éditeur

CEDEJ - Centre d'études et de documentation économiques juridiques et sociales

Édition imprimée

Date de publication : 30 septembre 1997

Pagination : 231-250

ISSN : 1110-5097

Référence électronique

Dina El-Khawaga, «Les droits de l'homme en Égypte », Égypte/Monde arabe [En ligne], 30-31 | 1997, mis en ligne le 08 juillet 2008, consulté le 07 juillet 2022. URL : http://journals.openedition.org/ema/ 1649 ; DOI : https://doi.org/10.4000/ema.1649

Ce document a été généré automatiquement le 7 juillet 2022

Tous droits réservés 


\title{
Les droits de l'homme en Égypte
}

\author{
Dynamiques de relocalisation d'une référence occidentale
}

\section{Dina El-Khawaga}

1 Dans les pages suivantes, il s'agira moins des visions de l'Occident comme entité objective ou imaginée que des droits de l'homme en tant que système de référence de provenance étrangère. Les raisons de ce glissement d'un objet «Occident » vers celui « d'une référence occidentale » sont multiples. Pour ne s'en tenir qu'aux plus saillantes, j'en citerai deux. La première est que le discours tenu explicitement sur l'Occident parmi les militants égyptiens des droits de l'homme ne se distingue en rien - au niveau du processus de construction, comme des genres produits - de l'ensemble des sens accordés à cet «autre » par les acteurs politiques égyptiens. Présentés variablement sous l'angle de l'altérité politique, de l'inégalité croissante entre le Nord et le Sud, ou de celui du conflit identitaire ${ }^{1}$, les "Occidents» des militants égyptiens des droits de l'homme rejoignent parfaitement le reste des représentations politiques à ce sujet. En d'autres termes, ni l'affiliation à une valeur universelle, ni la proximité effective avec des interlocuteurs gouvernementaux ou non-gouvernementaux occidentaux ne parviennent à produire - du moins au niveau du discours - des visions d'un ou de plusieurs « Occidents » qui seraient propres à nos acteurs des droits de l'homme.

2 La seconde raison découle directement de la première. Elle est liée à la nécessité de se déprendre de cette absence apparente de différenciation entre acteurs au sujet des «Occidents»: il s'agira donc de ne pas alimenter la thèse préétablie de la prédominance de l'idiome identitaire dans la construction de "l'autre occidental » (que cet idiome soit nationaliste, islamique ou autre), au sein des sociétés arabomusulmanes en général et de la société égyptienne en particulier. C'est afin de démanteler ce type de postulat qui se contente d'affirmer le consensus vraisemblable autour de l'altérité politique occidentale que j'ai choisi d'arrêter ma piste d'investigation sur les modalités de construction de sens d'une «référence occidentale » - celle, en l'occurrence, des droits de l'homme.

3 L'avantage d'une telle délimitation ne réside pas uniquement dans un parcellement, en plusieurs cibles plus précises, de l'objet insaisissable et totalisé qu'est l'Occident. Il réside surtout dans le remplacement d'un exercice épineux de répertoriage - celui du 
discours ou, au mieux, des conditions de sa production - par celui de la description d'une situation d'interaction. Autrement dit, il est question de déplacer l'attention du «dit» et des raisons (culturelles ou politiques) de son énonciation vers l'étude du contexte et des protagonistes qui interagissent au sein d'un espace collectif donné. Il s'agit donc de laisser de côté les énoncés des militants égyptiens des droits de l'homme au sujet de l'Occident et de décrire la triple action d'extraction, de ré-agencement, et de mobilisation d'une référence perçue comme exogène, au sein d'un contexte précis : le champ politique égyptien. Le choix d'une telle prise de vue nécessite cependant que l'on précise les termes constitutifs du cadre d'analyse proposé.

4 Vient d'abord la définition des acteurs, de leur système d'action et des champs d'action au sein desquels ils agissent. J'entends ici ceux qui s'auto-dénomment volontairement les «activistes des droits de l'homme » et qui agissent dans le cadre des organisations nationales, régionales, ou des centres d'études concernés par les droits de l'homme. Par système d'action j'entends ces opérations d'affrontement, d'articulation, voire d'imbrication de la référence des droits de l'homme - dite occidentale - et du reste des systèmes de référence supposés ancrés dans les représentations politiques de l'ensemble national. Par champ d'action, enfin, je n'entends pas uniquement l'espace régi par les activités concernant les droits de l'homme, mais l'univers d'échange entre nos acteurs et le reste de protagonistes locaux et non-locaux. Il faut dire que j'appelle l'ensemble de ce processus la relocalisation d'une référence et cela, pour souligner les modes de diffusion et de validation, entrepris par des acteurs précis (ici, les militants égyptiens des droits de l'homme), d'un code dont la provenance pose parfois problème dans l'interaction avec d'autres champs d'action. Autrement dit, il s'agit ici en gros du champ politique égyptien, structuré autour de partis politiques, de groupes idéologiques, et de mouvements identitaires.

5 En fait, bien qu'initialement inspirée par le terme de "délocalisation", forgé par Giddens (1990) pour décrire l'opération de transposition d'une valeur depuis la société qui l'a produite jusque dans d'autres univers n'ayant pas contribué à son élaboration, j'ai choisi délibérément de le remplacer par celui de "relocalisation » et cela pour plusieurs raisons. La première, et la plus évidente, provient du fait que la notion de délocalisation suppose implicitement un lien ombilical entre l'idée, la valeur, ou l'objet traversant des frontières et les localisations qui lui ont servi de cadre initial d'émergence ou de développement. Le problème que pose ce genre de postulat, c'est qu'il parvient à instaurer ce que Jean Leca dénonce lorsqu'il dit que « la construction du monde universel-occidental... est toujours suspectée d'établir, ou de défendre, non pas la Loi, mais sa loi présentée comme la Loi » (Leca, 1990). Cela ne reviendrait pas seulement à dire que tout ce qui peut être labellisé comme universel est foncièrement occidental - qu'il s'agisse de la modernité, du nationalisme, de la valeur de la liberté, ou de l'industrialisation -, mais à véhiculer une confusion grandissante entre les références et valeurs et les localisations qui prétendent à l'universalité en s'érigeant comme des modèles à suivre. Cette vision occidentalo-centrée des processus de diffusion ou de partage des valeurs et des objets masque donc la multiplicité des formes d'immixtion anciennes et/ou nouvelles qui ont pu se manifester tout au long des deux derniers siècles et qui ont contribué à tracer des lignes - ne serait-ce que fluides - de démarcation entre un patrimoine commun puisque universel et les formes occidentales de sa particularisation. La seconde raison est que la délocalisation comme processus tend à sur focaliser l'attention sur l'opération d'extraction multiforme et décentrée d'une référence, et cela au détriment de celle de sa réinsertion au sein d'un champ 
donné. Les trajectoires réservées aux références extirpées en dehors de leurs frontières initiales deviennent ainsi le fil conducteur de toute lecture des conséquences de la modernité et non la riche diversité des formes successives d'association, de dissociation, et de réassociation que des acteurs entreprennent au cours d'un processus qui engage plusieurs localisations à la fois sans toutefois être totalement tributaire de l'une d'entre elles.

6 Pour clore cette introduction, notons enfin qu'en délimitant de telle manière mon objet d'étude, je suis loin de postuler que la «relocalisation des droits de l'homme » est une affaire strictement interne (c'est-à-dire un processus d'endogénéisation du sens), autrement dit, en rupture totale avec l'environnement transnational. Ce que je veux illustrer, c'est que le processus de relocalisation, en tant qu'effort de "recadrage", dépend à la fois des systèmes de valeurs admis au sein d'une société, du type d'interaction mis en œuvre entre des acteurs qui recourent à différents systèmes de référence, et des entreprises diverses de compatibilisation de l'endogène et de l'exogène engagées par ces derniers.

7 Identifions maintenant les mécanismes constitutifs de ce processus de relocalisation. Trois opérations imbriquées y apparaissent: l'encodage du principe des droits de l'homme dans le vocabulaire politique égyptien, son homologation par rapport à l'intérieur, c'est-à-dire, sa redéfinition à la lumière des autres références reconnues et admises par l'ensemble des acteurs politiques nationaux ou régionaux comme étant les leurs (nationalisme, ré-islamisation, justice sociale, panarabisme), et enfin, son homologation par rapport à l'extérieur, c'est-à-dire son réagencement par rapport aux normes érigées par les défenseurs de ce principe, dans un ailleurs variablement défini mais toujours identifiable, et qui se présentent tantôt comme fondateurs, tantôt comme protecteurs des droits de l'homme un peu partout dans le monde. Mais avant de décrire ces trois opérations, il importe d'ouvrir une parenthèse pour identifier les acteurs et les champs d'action impliqués dans ces opérations. Celle-ci me semble indispensable non seulement pour contextualiser ma lecture mais aussi pour illustrer à quel point la relocalisation, dans une situation de transnationalisation, va instituer une multiplicité d'acceptions de la référence mobilisée, au sein même d'une seule localisation et selon le type d'interactions engagées.

\section{Qui sont ces activistes des droits de l'homme?}

Pour synthétiser, je présenterai l'ensemble des acteurs des droits de l'homme en termes de mouvance et non de mouvement. Cela, en raison des grandes divergences qui existent entre ces derniers et qui ne les empêchent pas pour autant d'assurer des formes constantes de coopération et de coordination de leurs actions. J'emploie la notion de mouvance également pour souligner un autre fait : celui de l'alternance de la centralité entre divers groupes d'acteurs agissant tous au nom des droits de l'homme mais leur accordant des significations dissemblables. Une alternance que je dégage événementiellement en trois phases successives et que j'appellerai, dans l'ordre, le lancement politicien, la radicalisation et la diversification. 


\section{Le lancement politicien}

Cette première phase commence avec la fondation de l'Organisation arabe des droits de l'homme (OADH) à Chypre en 1983. C'est à l'initiative d'une bonne centaine de personnages publics et d'intellectuels célèbres du Moyen-Orient pour la plupart, qu'une rencontre officielle est organisée et qu'une demande de reconnaissance régionale est formulée ${ }^{2}$. Le rôle des protagonistes égyptiens est majeur dans le lancement de l'entreprise ${ }^{3}$, non en raison de leur sensibilité ou expertise dans ce domaine, mais parce que toute une frange de ces acteurs entend exercer un rôle d'experts ou de conseillers vis-à-vis du gouvernement égyptien, en dressant "la transition vers la démocratie » comme sa nouvelle source de légitimité. La référence aux droits de l'homme semble alors plus qu'appropriée aux yeux de cette foule d'acteurs issus des formations politiques de la libération nationale ou de celles, idéologiques, du développementalisme. Grâce à cette nouvelle cause, le militantisme semble, d'une part, leur garantir une position éventuelle, non pas de technocrates, mais de conseillers susceptibles de délimiter les nouvelles modalités d'action de la «légitimité démocratique ». L'adhésion à la valeur des droits de l'homme leur permet, d'autre part, de réactualiser les idiomes politiques adoptés ou hérités des années soixante et soixante-dix et qui semblent dépassés, vu les changements nationaux et internationaux qui voient le jour dès le début des années quatre-vingt. Le lancement d'une organisation régionale et non simplement nationale leur sert, enfin, à demeurer apparemment fidèles à leurs orientations panarabes antérieures, tout en constituant une carte de pression valable sur chacun des pouvoirs politiques de la région, à commencer par l'Égypte.

\section{La radicalisation}

10 Cette dimension politicienne, voire élitiste, ne tarde pas cependant à céder la place à une seconde configuration, celle de la radicalisation. Cette seconde phase commence avec l'échec manifeste des tentatives de l'OADH à se faire admettre comme interlocuteur politique valable et à acquérir un statut associatif stable sur le double plan régional et national ${ }^{4}$. Cela pousse une bonne partie des instigateurs de la première phase, soit à modérer leurs ambitions, soit à se tourner vers d'autres domaines d'actions. C'est à partir de ce moment (qui se situe entre 1986 et 1989) que la mouvance égyptienne des droits de l'homme assiste, d'une part, à une " domestication politique » croissante de l'OADH et se voit investie, d'autre part, par des éléments radicaux, nettement plus jeunes et ostensiblement moins concernés par la coordination avec le pouvoir politique égyptien. Marxistes, communistes, nassériens mais aussi nationalistes du Wafd, dont le dénominateur commun réside dans un désenchantement politique profond vis-à-vis de leurs partis respectifs comme vis-à-vis du processus de démocratisation, vont alors activer l'organisation égyptienne des droits de l'homme, l'OEDH, légalement maintenue sous la forme précaire d'organisation en voie de constitution mais reconnue comme antenne de l'organisation régionale.

11 Deux dimensions paraissent dès lors centrales dans la structuration de la mouvance des droits de l'homme: la critique du pouvoir politique et l'usage des droits de l'homme comme une référence quasi idéologique (c'est-à-dire ayant une capacité d'interprétation totalisante et une portée morale et missionnaire). C'est dans ce glissement d'un cadre revendiquant la démocratisation à partir des pouvoirs politiques 
vers un autre, militant pour une démocratisation «à partir du bas », que la médiatisation du principe des droits de l'homme va prendre une nouvelle tournure: celle qui en fait l'épine dorsale du renforcement de la société civile et une des garanties indispensables de son affirmation face aux restrictions juridiques et politiques dressées par le gouvernement égyptien et, dira-t-on souvent, "ses lois d'exception". L'abondance des enquêtes menées sur les atteintes aux libertés politiques (arrestations, torture, etc.) constituera ainsi la tâche principale des militants de l'OEDH entre 1988 et 1992 et donnera lieu à de nombreux rapports dévoilant les pratiques répressives du pouvoir politique vis-à-vis de l'ensemble de ses opposants politiques (marxistes, nassériens, mais surtout islamistes).

12 Cette mobilisation aux niveaux national et international ne va pas tarder à opposer farouchement les activistes des droits de l'homme appartenant à l'OEDH et les membres de l'Establishment qui affublent ces derniers de l'image de «fauteurs de troubles » qu'il convient de mater ou, du moins, de marginaliser ${ }^{5}$. De nombreuses mesures sont alors prises dans cette perspective qui va de l'occultation totale de l'OEDH comme organisation jouissant d'une existence légale à l'arrestation effective de certains de ses porte-parole ${ }^{6}$, en passant par des menaces de liquidation ${ }^{7}$. En outre, une large campagne médiatique visant à discréditer politiquement et moralement les activistes des droits de l'homme est menée afin de stigmatiser l'ensemble de la mouvance égyptienne. Il serait cependant réducteur de présenter ce conflit comme étant binaire. Très rapidement, il devient tripolaire dans la mesure où les militants des droits de l'homme comme le pouvoir égyptien vont recourir à l'Occident pour valider leurs prises de positions antagoniques. Pour les premiers, les organisations occidentales et/ ou internationales feront figure d'arbitres ou du moins d'instance potentielle de pression internationale contre le pouvoir politique égyptien et ses transgressions des règles universelles des droits de l'homme. Pour le second, le renforcement des alliances stratégiques avec l'Occident constituera une garantie permettant la mise en sourdine épisodique de tout ce dossier. Cette forme d'interférence du transnational dans l'opposition pouvoir politique/ mouvance égyptienne des droits de l'homme ne sera pourtant qu'une parmi toutes celles qui verront le jour au cours de la phase suivante.

\section{La diversification}

13 L'activité centrale de la mouvance égyptienne, focalisée sur le répertoriage et la publication des atteintes aux libertés politiques, engage des ex-militants politiques sur un nouveau terrain d'activité qu'ils maîtrisent mal au départ. Soucieux de se forger une crédibilité hors de leurs frontières nationales et de s'instituer comme protagoniste valable au niveau du mouvement international des droits de l'homme, les activistes égyptiens vont alors commencer à centrer leur attention sur l'étude des protocoles internationaux qui définissent les étapes de l'enquête, les programmes d'entraînement, l'action à mener d'abord auprès des appareils étatiques nationaux, les formes et les limites de l'assistance juridique ou médiatique, la rédaction des rapports officiels ainsi que le repérage des institutions destinataires. Cela les expose à un nouvel univers d'échange régulier de vues avec les organisations occidentales et internationales, qui tiennent leurs standards pour universels et communs à tous les mouvements des droits de l'homme, au Nord comme au Sud. À partir de là, la mouvance des droits de l'homme va progressivement adjoindre d'autres volets à ses activités de répertoriage des transgressions étatiques des libertés politiques: ainsi de l'assistance juridique aux 
citoyens démunis devant leurs droits civiques bafoués, de la conscientisation populaire eu égard aux droits constitutionnels et légaux, de la recherche scientifique dans ses deux versions académique et vulgarisée et du ciblage de certaines catégories sociales, définies comme étant plus exposées aux transgressions de leurs droits (femmes, enfants, citoyens coptes).

14 L'élargissement des thèmes à couvrir et du public ciblé émousse graduellement l'aspect militariste de l'OEDH qui a prévalu lors de la seconde phase et cède la place à une nouvelle configuration qui dépasse l'anti-étatisme et les modes idéologiques de dénonciation du pouvoir en place. Ainsi, la multiplication des contacts avec les instances inter et trans-nationales concernées par la défense des droits de l'homme ne pondère pas uniquement l'opposition des militants affiliés à ce mouvement et de l'État égyptien, mais elle les " professionnalise $»^{8}$ aussi et les sensibilise aux nouveaux débats internationaux sur les libertés religieuses, le code de la nationalité, les droits de la femme, ou des minorités culturelles, voire sociales, et donne ainsi lieu à un nouveau type de militantisme d'apparence plus universaliste qu'oppositionnel.

Cette diversification des interventions dans des domaines moins politiques ouvre par ailleurs la voie à de nombreuses controverses au sein même de l'OEDH, voire de l'ensemble de la mouvance, autour des risques d'altération que suscite une telle ouverture sur le mouvement transnational des droits de l'homme. Des polémiques en tout genre émergent alors et commencent à scinder la mouvance en plusieurs courants quasi antagoniques. Certains militants récusent le principe même de financement occidental et démissionnent de l'OEDH ou d'autres organisations non gouvernementales en raison de l'aide financière ou technique fournie par des organismes occidentaux, et qualifient publiquement les nouvelles options de risque majeur de dépendance politique envers l'Occident ${ }^{9}$. D'autres, en revanche, affichent leur agacement devant les accusations dressées contre la mouvance et préfèrent déplacer leurs activités vers les nouveaux réseaux de militantisme civil de type local, appelé en Égypte " travail non gouvernemental » (al -'amal al-ahlî), et qui se développe indépendamment des formations idéologiques et de leurs représentants. Certains, enfin, se réfugient dans la branche la moins controversée de l'activisme des droits de l'homme, celle de la recherche scientifique.

C'est à la lumière de telles altercations que l'on parvient à expliquer la constance, ainsi que la violence, de la campagne médiatique animée par les militants des droits de l'homme au sujet de la prééminence de l'agenda national sur l'agenda international, de la légitimité du recours à une aide non arabe en matière de finance ou de savoir-faire, enfin, de la corruption éthique et politique d'une partie de la mouvance. C'est dans ce cadre qu'on parvient aussi à expliquer l'ampleur du phénomène des « dissidences » des responsables des deux grandes organisations, et qui donneront lieu, à partir de 1992, à un foisonnement de centres d'études privés dit "spécialisés » dans le répertoriage, la pédagogie ou l'assistance juridique ; les droits de la femme, de l'enfant, ou des victimes de la violence non gouvernementale (c'est-à-dire islamiste). Ce processus de diversification, connu en Égypte sous la forme de la spécialisation et tenu pour l'un des signes les plus positifs de la "maturation" de la mouvance selon les critères transnationaux, laisse voir en même temps un phénomène de division chez les activistes des droits de l'homme, empêtrés dans leurs controverses internes. Et l'Establishment de s'en saisir comme d'un argument visant à discréditer ces activistes 
que l'on présente dès lors comme des opportunistes politiques vivant aux dépens des financements étrangers ou des agents d'un Occident corrupteur.

\section{Les mécanismes de relocalisation}

17 Je ne me suis pas attardée sur ce brossage de la mouvance des droits de l'homme à des fins purement sociographiques. À travers la périodisation esquissée, j'entendais faire surgir, d'une part, le constant réagencement du type d'acteurs, d'enjeux, et de contraintes au sein de la mouvance des droits de l'homme, et souligner, d'autre part, la gamme des formes d'imbrication du local et du transnational et qui va de la mobilisation instrumentale d'une référence à la «conversion » quasi totale de certains militants - auparavant nationalistes, marxistes ou nassériens - à cette référence qualifiée d'universelle. Je tenais aussi à mettre l'accent sur la cristallisation progressive de nombreux pôles définissant différemment la référence et les modalités d'action à suivre. Je voulais enfin insister sur le fait que la genèse de la mouvance ne dépendait pas totalement de i'intentionnalité des acteurs mais d'un certain nombre d'interactions internes et externes ${ }^{10}$. À la lumière de ces postulats, je vais à la fois définir chacune des opérations constitutives du processus de la relocalisation et donner des exemples à partir de mon terrain. Aussi faut-il rappeler que celles-ci ne correspondent qu'approximativement au principe de périodisation esquissé auparavant et qu'elles se manifestent d'une façon plutôt simultanée, d'où l'intérêt de les décrire comme des opérations imbriquées.

\section{L'encodage}

L'encodage, comme action individuelle ou collective d'extraction d'une référence ou d'un objet de son univers initial et son insertion dans un cadre cognitif qui n'a pas participé à sa production, implique: $1^{\circ}$ ) que soit présumée une capacité du cadre récepteur à accorder des acceptions ou des usages qui ne dépendent que des structures et des catégories internes $; 2^{\circ}$ ) que soit postulée une nécessaire différence, voire une rupture, entre l'usage de départ et l'usage d'arrivée. Cette définition né pose pas de problème pour peu que soient remplies trois conditions :

1. qu'on ne postule pas que ces cadres récepteurs reflètent une culture pure ou préétablie mais qu'on y voie plutôt de nombreuses structures subjectives au sein desquelles s'enchevêtrent aussi bien éléments locaux qu'éléments moins locaux, «routinisés » à travers un long processus historique qui n'atteint jamais son terme;

2. qu'on s'abstienne d'affirmer la présence d'un unique code au sein d'une localisation et qu'on accepte que cette dernière se compose d'une diversité de systèmes d'action croisés selon les groupes et les références qu'ils défendent;

3. qu'on admette que cette opération d'encodage d'une référence n'exclue pas l'entreprise d'autres stratégies cognitives qui ne visent pas principalement la ré-insertion d'un « objetsens » comme objectif ultime.

L'exemple de la mouvance égyptienne des droits de l'homme est révélateur à ce propos, dans la mesure où l'on repère, dès la première phase, un usage qu'on pourrait qualifier d'instrumental du principe des droits de l'homme à des fins plutôt politiciennes (exemples : renouveler les idiomes d'une élite politique marginalisée ou pousser le pouvoir politique à une coopération) - usage qui persisterait jusqu'aujourd'hui parmi 
les activistes de l'OADH. Mais au-delà d'une analyse stratégique, il paraît évident que cette première modalité d'insertion de la valeur des droits de l'homme se fait, ici, selon les mêmes schémas qui ont prévalu dans les années soixante et soixante-dix, au niveau des modes de validation de l'affiliation à la valeur du non-alignement, à celui de l'arabisation des idées socialistes, à celui des idiomes de la planification centralisatrice, etc. ; c'est-à-dire, en faisant appel à une mobilisation panarabe des intellectuels et des hommes politiques pour fonder idéologiquement la légitimité, voire la nécessité, du processus d'insertion en cours et en s'adressant exclusivement aux pouvoirs politiques en place, pour asseoir cette valeur en l'intégrant dans leurs cadres référentiels.

L'on assiste, en outre, surtout à partir de la seconde phase, à une insertion de la même référence pour nourrir la dichotomie radicale État/société civile, qui, en tant que catégorie référentielle, est initialement tout aussi exogène que les droits de l'homme, mais qui a été intégrée et intériorisée antérieurement par un ensemble d'acteurs politiques se revendiquant comme opposants politiques au pouvoir en place et à ses options. Et alors que la première forme d'encodage prend corps au sein de l'idiome de la libération nationale (renforcement de la libération de la patrie et de ses citoyens face à l'obscurantisme et au sous-développement, Limasol-Chypre, 1983), la seconde apparaît en termes de revendication d'un État de droit et d'affirmation de la société civile, d'urgence de la réforme juridique, du respect de l'ensemble des droits civiques (fonder des partis, associations, organes de presse, mais aussi pouvoir recourir aux grèves ou aux manifestations). Ici, on constate à quel point les idiomes politiques locaux sont loin d'exprimer synchroniquement ou diachroniquement ${ }^{11}$ un cadre unifié. On voit aussi à quel point le type d'acteurs inscrits dans une localisation donnée est loin d'être homogène et encode la référence en question, par conséquence, dans des structures diversifiées de sens, toutes invariablement perçues comme locales, alors qu'elles ne le sont pas - étymologiquement du moins.

\section{L'homologation par rapport au local}

Comme opération, cette forme d'homologation diffère de l'encodage en ce sens qu'elle ne vise pas une réappropriation de la référence au sein d'une localisation mais une reconnaissance, voire une admission de celle-ci par des acteurs mobilisant d'autres référentialités. C'est plutôt une recherche de "compatibilité » de la valeur relocalisée avec les autres catégories de sens. Aussi faut-il rappeler qu'aucun ordre chronologique ni logique ne régit le rapport de l'encodage et de l'homologation avec le local. C'est davantage la simultanéité, voire "l'éclectisme » qui caractérise le recours des acteurs à telle ou telle opération. Dans la perspective d'homologation, des opérations de suppression, d'adjonction, ou de reformulation de la référence des droits de l'homme sont alors entreprises afin d'identifier des terrains de convergence ou de divergence avec les catégories référentielles en cours dans le champ d'action. Ce processus d'homologation peut prendre cependant plusieurs formes qu'il ne faut ni confondre, ni perdre de vue.

La première est l'effort de saper toute controverse référentielle en termes d'endogénéité ou extranéité, à partir de l'affirmation que les catégories référentielles dites endogènes contiennent de facto le pendant objectif de la valeur universelle à homologuer. C'est à la lumière d'une telle définition opérationnelle qu'on peut expliquer le foisonnement d'écrits et de pratiques discursives parmi les activistes des 
droits de l'homme au sujet de la parfaite compatibilité entre L'Islam et les droits de l'homme (Al-Sayyed Saïd, 1996), la vénération réservée aux Droits de l'enfant dans la culture arabo-musulmane (Mannâ $\left.\hat{a}^{1}, 1996\right)$, ou la centrante de la lutte pour le respect des Droits de l'homme dans la continuité du conflit Nord/Sud ${ }^{12}$. Ici, les auteurs de telles contributions - mais aussi d'une bonne centaine d'autres -s'accordent tous à véhiculer un même message malgré la diversité de leurs destinataires: les défenseurs de la référence islamique, nationaliste ou tiers-mondiste portent en eux - de par leurs propres référentialités - les bases d'un patrimoine universel commun dont la valeur des droits de l'homme présente une partie intégrante. Autrement dit, ils militent de fait pour la validation de cette valeur même s'ils ne le déclarent pas explicitement.

Outre donc la diffusion, à travers des idiomes plus familiers, des idées des droits de l'homme au grand public égyptien, ou l'affirmation de la diversité des voies d'affiliation à cette valeur universelle, ce qui est recherché ici, c'est la mise en place d'un débat susceptible d'identifier des terrains partiels d'entente, en lieu et place d'échanges polémiques - ceux d'acteurs engagés dans la validation, en termes d'authenticité ou d'extranéité, de référentialités différentes. Cet intérêt à forger des espaces de compatibilité illustre la nécessité de l'homologation par rapport aux autres références dites locales et qui sont, dans l'exemple égyptien, comme je l'ai dit auparavant, le nationalisme et les fondements culturels et politiques islamiques, le panarabisme, la justice sociale, le tiers-mondisme, etc. Il faut ajouter que la production engagée sur cette voie comporte un volet central dans les activités et les pratiques discursives de la mouvance des droits de l'homme et qu'il est difficile de trouver une publication, une rencontre ou une déclaration qui n'inclue pas cette première forme de tentative de compatibilisation ${ }^{13}$.

Vient ensuite une seconde forme, non moins fréquente, quoique plus indirecte, d'homologation par rapport au local : celle qui consiste à établir un lien de causalité entre des maux ou des crises politiques, dont souffre la société égyptienne au présent, et la faiblesse du mouvement égyptien ou arabe des droits de l'homme. Ainsi, l'arriération de l'Égypte ${ }^{14}$, les problèmes de transition vers la démocratie ${ }^{15}$, la crise du développement ${ }^{16}$, la question de la citoyenneté manquée ${ }^{17}$ ou des menaces pesant sur la liberté de la presse (Al-Sayyed Saïd, Hassan, 1995), sont-elles les conséquences d'une même raison initiale : celle du non-ancrage et du non-respect des valeurs des droits de l'homme au sein de la société égyptienne, ou, plus généralement, des sociétés arabes.

Cette articulation des enjeux collectifs et des droits de l'homme constitue une seconde voie d'enracinement de cette référentialité au sein de l'espace public égyptien; une articulation qu'on ne peut qualifier d'encodage dans la mesure où la valeur à homologuer n'est pas simplement insérée dans un paradigme d'action plus large, mais est instrumentalisée comme perspective d'explication complémentaire de certains problèmes qui semblent disparates mais qui s'entrelacent à partir de ce type de lecture. Il faut dire que cette forme d'action cognitive, qui fait partie de l'opération d'homologation par rapport au local, et qui s'appuie sur l'établissement d'un lien de causalité entre des problèmes et une valeur supposée faire défaut au sein d'une collectivité, est loin de présenter un exercice politique ou intellectuel inédit en Égypte. De nombreux auteurs ont montré comment certains acteurs de la réforme de l'Égypte de la première moitié du $\mathrm{XX}^{\mathrm{e}}$ siècle avaient procédé de la même manière pour valider la nécessité, voire l'urgence, d'un nombre de valeurs dites exogènes, telles l'éducation moderne, l'émancipation des femmes, la discipline ou la propreté, et avaient choisi 
quasiment le même type de titres d'ouvrages dans leurs opérations de diffusion et de validation des valeurs qu'ils défendaient (Roussillon, 1995). Ce détour par l'analogie historique permet de se déprendre d'un stéréotype assez omniprésent à propos des entreprises des acteurs face à l'articulation endogène/exogène et qui consiste à postuler que ces tentatives d'imbrication hybrides et multiformes sont, intrinsèquement, un produit récent de la mondialisation. Il permet d'illustrer empiriquement "la profondeur historique ", pour paraphraser A. Abdel-Malek, de ce type d'opérations cognitives au sein de l'espace public égyptien.

Suit enfin une troisième forme d'homologation par rapport au local. Plus allégorique, celle-ci se manifeste à travers l'émission d'un certain nombre de signes qui cherchent tous à « indexer » (Ferrié, 1996, p. 15 à 23) des symboles arabo-islamiques aux pratiques des militants égyptiens des droits de l'homme. L'appellation du périodique le plus sérieux des droits de l'homme en Égypte, Riwâq 'Arabî, présente un premier exemple de cet exercice d'indexation dans la mesure où le terme « Riwâq » renvoie à l'entrée d'une mosquée et d'une école coranique. L'annexion de l'attribut « 'Arabî» a ici pour objectif non d'indexer un symbole d'une provenance autre - ethnique et non religieuse - mais de renforcer la métaphore $d u$ « rassemblement pour amorcer une action bénie telle que la prière ou l'apprentissage du Coran", en s'appuyant sur une image historique supposée commune à tous puis qu'ancrée dans le patrimoine arabo-islamique. Le Salon d'Ibn Rushd, surnom accordé au séminaire mensuel portant sur les droits de l'homme, exprime la même logique. Ici, l'indexation ne se fait pas à travers le rattachement d'une activité éditoriale actuelle à une forme historique de socialisation religieuse, mais à partir de la réappropriation d'une figure célèbre du patrimoine arabo-islamique, le philosophe Averroès. En l'érigeant en hôte fictif, les militants des droits de l'homme associent leurs rencontres à tout un courant de pensée historiquement perçu comme réformateur et critique tout en demeurant légitime du point de vue de l'orthodoxie sunnite. Ce faisant, ils parviennent à véhiculer une homologie implicite entre un passé arabo-islamique et un présent national ; entre Ibn Rushd et ses disciples, et une branche de la mouvance actuelle des droits de l'homme. Tout emblématique qu'il soit, ce type d'exercices disparates d'indexation de sens arabo-islamique ne présente pas d'opérations cérémoniales ou vides de sens. Il illustre un niveau des modalités de rattachement du local et de l'exogène, de l'ancré historiquement et de l'importé au présent, qu'il convient de prendre en compte pour délimiter la diversité des opérations constitutives du processus de la relocalisation.

\section{L'homologation par rapport à l'exogène}

Pour définir opératoirement ce troisième type d'homologation, je distinguerai l'homologation par rapport à l'intérieur de celle engagée par rapport à l'extérieur selon trois modalités. La première implique que toute homologation par rapport à l'extérieur, dans le sens d'un effort de standardisation avec l'exogène, exige d'abord un degré de relocalisation de la référence mobilisée ; autrement dit, une forme minimale de réappropriation permettant aux acteurs de prétendre posséder un usage particulier de la référence initialement inscrite dans un cadre universel ${ }^{18}$. À la différence donc de l'encodage et de l'homologation par rapport à l'intérieur, l'homologation par rapport à l'extérieur n'est engagée comme opération cognitive que d'une façon temporellement décalée. La seconde, évidente, concerne la diversité des instances visées par cette opération; autrement dit, la fluidité des modes d'identification du transnational, ainsi 
que de ses «standards ", lors de l'entreprise d'homologation par rapport à ce dernier. La troisième suppose que cette opération - en tant que situation d'interaction - puisse tout aussi bien engager des protagonistes locaux au sujet de la marge de standardisation d'un ou de plusieurs objets qu'ils perçoivent comme les leurs que mobiliser des acteurs internes et externes autour d'un débat - réel ou feint - à propos des modes de "comparabilité » des valeurs ou objets avant, pendant et après leur relocalisation. Autrement dit, contrairement aux opérations précédentes, l'homologation par rapport à l'exogène présente une interaction à protagonistes variables.

En ce qui concerne le premier point, la trajectoire de la mouvance égyptienne des droits de l'homme révèle effectivement un léger décalage entre les deux premières opérations et cette dernière. En fait, la production d'un discours affirmé sur la "spécificité » du mouvement arabe des droits de l'homme soulignant à la fois les douloureuses conditions, légales et politiques, imposées par les pouvoirs en place et l'indifférence ${ }^{19}$, voire l'opportunisme des acteurs politiques à son égard, devient une idée-force de la mouvance à partir des années quatre-vingt-dix pour insister sur la différence des trajectoires du mouvement universel des droits de l'homme, ancré et respecté partout dans le monde, et le mouvement égyptien ou arabe, persécuté et constamment instrumentale par la classe politique. Là, le rappel constant de la spécificité ne se fait pas en vue d'une démarcation identitaire ou politique. Au contraire, c'est pour souligner les difficultés qui régissent les tentatives de participation locale ou régionale à une démarche universelle que les activistes égyptiens entreprennent ce type d'opération de comparabilité. De même, l'on peut citer l'ampleur d'un discours, parmi certains militants, qui insiste sur la difficulté de militer pour la valeur des droits de l'homme dans des sociétés fortement mobilisées autour du réfèrent religieux - discours qui, pour autant, ne prend pas partie dans cette mobilisation. Ce discours qui n'émerge qu'à partir de 1992, soit à partir de l'acquisition, par la mouvance, d'une certaine crédibilité politique au sujet de son rapport avec la mouvance des islamistes, tend également à mettre l'accent sur des "contraintes ou épreuves» qui entravent la standardisation ${ }^{20}$. Enfin, l'appel récent à la "spécialisation » dans telle ou telle branche d'activisme - s'alignant sur la trajectoire transnationale du mouvement et se manifestant de plus en plus comme ligne interne de démarcation au sein de la mouvance égyptienne - ne voit le jour que depuis trois ou quatre ans, suite à l'ouverture massive sur les partenaires occidentaux.

Dans tous ces cas de figures, les militants égyptiens des droits de l'homme tentent de définir leur entreprise et leurs options - consensuelles ou divergentes -à la lumière de ce qui se fait ailleurs. Leur souci dépasse le simple maintien d'une spécificité creuse ou la justification de l'adhésion inconditionnelle à une forme préétablie d'action. Ce qu'ils tentent de construire dans ces exemples précis, c'est la "comparabilité » entre un usage particulier qui est le leur et des usages provenant d'ailleurs qui peuvent faire figure tout aussi bien de repères que de repoussoirs. Il est évident que ce type d'exercice cognitif à propos du militantisme au nom des droits de l'homme est assez inédit. Auparavant, soit durant les phases de lancement et de radicalisation, l'évocation de la dimension transnationale du mouvement des droits de l'homme se faisait, parmi les activistes égyptiens, soit furtivement, soit dans les termes de l'altérité politique et identitaire; autrement dit, de l'urgence de contribuer au nom de l'arabité, ou du nationalisme égyptien au mouvement universel se rapportant à cette valeur. 
30 Au sujet de l'identification multiforme du transnational, l'on constate facilement à quel point les stratégies visant à situer l'expérience égyptienne ou arabe dans le cadre dit transnational des droits de l'homme évoquent une multiplicité, soit de standards, soit de protagonistes, suivant les situations d'interaction et visent, par cette diversité, un extérieur variablement défini selon les circonstances, les enjeux, etc., afin d'entreprendre l'opération d'homologation. Ainsi, se profilent de nombreuses modalités d'identification de ce qui constitue le standard dans sa double acception de contrainte ou de suggestion. Dans ce cadre, vient d'abord la dichotomie entre le transnational comme catégorie référentielle et l'universel comme niveau irréversible d'appartenance. Apparaît ensuite l'opposition au sein des conventions universelles ratifiées .par les États membres de l'Onu - conventions qui opèrent comme référentialité englobante, tout en étant spatialement et temporellement situées. Suivent enfin les actions et stratégies (connues sous le label d'agenda) perçues tantôt comme standards implicitement contraignants ou comme forme de particularisation occidentale d'un principe universel que le monde entier partage à travers une multiplicité d'agendas construits.

31 Quant à l'identification de l'extérieur comme pôle émetteur d'un standard, un non moindre foisonnement de modalités d'identification émerge pour lui accorder soit le sens d'un cadre englobant soit celui d'un protagoniste extranational totalisé. Ainsi, il est tantôt identifié à l'expérience historique de l'Occident et de sa lutte laborieuse pour faire respecter les droits de l'homme, tantôt assimilé aux organisations internationales, présentées comme à la fois officielles et impuissantes mais légalement reconnues comme porte-parole du mouvement universel (Onu, Unesco, etc.). D'un autre côté, Il peut renvoyer aux gouvernements occidentaux, hypocrites en ce qu'ils appliquent la politique du «deux poids, deux mesures » eu égard aux droits de l'homme arabe ou palestinien ${ }^{21}$. Il peut renvoyer enfin à l'ensemble des organisations concernées par les droits de l'homme, indépendantes de leurs gouvernements et soucieuses d'encourager la coopération entre les militants du Nord et du Sud ${ }^{22}$. J'évoque ce point pour souligner l'énorme multiplicité des cercles de pertinence au niveau de l'homologation par rapport à l'exogène, et, en conséquence, l'ample diversité des genres produits par ce biais à propos de ce que l'on entend par exogène, transnational, ou universel.

Cela nous conduit au troisième point, celui de la variété considérable des protagonistes éventuels de ces opérations de standardisation. En effet, dans l'ensemble des exemples précédents tirés des publications, de l'observation directe ou des entretiens avec les militants égyptiens, l'on retrouve trois formes distinctes d'interaction en vue d'une homologation du local et du transnational. La première, la plus fréquente, est celle qui engage des militants égyptiens en désaccord sur telle ou telle mesure à prendre au nom de la défense des droits de l'homme : chacun y mobilise sa propre acception de ce qu'est le standard et désigne par implication un pôle exogène précis en vue d'échafauder son parti pris. La seconde est celle souvent intentée au nom du «redressement du mouvement égyptien des droits de l'homme ", non pas nécessairement lors d'une controverse, mais dans une perspective d'introspection ou d'auto-critique de tel militant ou groupe de militants, affilié à la mouvance. La troisième est celle mettant aux prises certains acteurs égyptiens et des représentants étrangers d'organisations internationales ou non gouvernementales occidentales, lors de rencontres ou de pourparlers techniques, politiques ou financiers, au sujet des priorités, des plans d'action, des prises de position, etc. Dans l'ensemble de ces interactions fort 
diversifiées, la logique adoptée par nos acteurs demeure la même : établir un mode de comparabilité avec ce qui est perçu comme le standard, à la lumière de l'acception accordée à la référence des droits de l'homme et ses supposés défenseurs exogènes.

En abordant ces trois opérations et les variantes que chacune d'entre elles inclut, je cherchais à montrer un aspect souvent omis des modalités de construction de sens d'une référence dite "occidentale " au sein d'un champ d'action qui ne l'est pas. Je voulais fournir une grille de lecture qui brise le stéréotype présentant le local et le transnational comme deux mondes possédant des logiques entièrement séparées, voire antagoniques. Je souhaitais aussi souligner à quel point la délimitation des frontières $\mathrm{du}$ local et de l'exogène est loin de constituer un mode de distinction stable ou défini une fois pour toutes. J'espérais également participer au débat portant sur la dichotomie encodage/homologation (Ferrie, 1996) à partir d'un terrain concret. Dans cette perspective, l'identification de trois opérations constitutives de la relocalisation ne cherchait pas tant à atteindre une formule conciliante face à cette dichotomie que de proposer une construction qui prenne en compte à la fois l'apport de ces notions opératoires et l'expérience d'un terrain riche de significations. La relocalisation comme grille de lecture nous permet ainsi de frayer un site d'observation, loin des discours d'acteurs surchargés de valorisation identitaire, qui illustre un cas concret d'enchevêtrement des systèmes de sens présentés trop facilement comme incompatibles dans les rhétoriques politiques alors qu'il n'en est rien dans les actions entreprises. Aussi nous permet-elle de nous situer loin des débats abstraits décryptant le rapport mouvant entre l'endogène et l'exogène, dans une conjoncture de mondialisation des «objets-sens ", où l'endogénéisation est «multiréférencée » et l'homogénéisation planétaire.

\section{BIBLIOGRAPHIE}

FERRIÉ J.-N., 1996, « L'appartenance des objets : problèmes d'anthropologie de la culture et de l'identité », Égypte/Monde arabe, $\mathrm{n}^{\circ} 25$.

GIDDENS A., 1990, The Consequences of Modernity, Stanford, Stanford University

Press.

Huqûq al-insân wa ta'akhkhurMisr (Les droits de l'homme et l'arriération de l'Égypte), 1993, Le Caire, éd. du Centre d'études et d'information juridiques pour les droits de l'homme.

MANNÂ' H., 1996, « Huqûq al-tjfl fi-l-thaqâfa al-'arabiyya al-islâmiyya » (Les droits de l'enfant dans la culture arabo-islamique), Riwâq 'Arabî, n 2, Le Caire, éd. du Centre du Caire pour les droits de l'homme.

ROUSSILLON A., 1995, « Réforme sociale et production des classes moyennes : Muhammad 'Umaret l'arriération des Égyptiens ", dans Entre réforme sociale et mouvement national : identité et modernisation en Égypte (1882-1962), Le Caire, Cedej. 
AL-SAYYED SAÏD M., 1996, « Al-lsiâm wa huqûq al-insân » (L'islam et les droits de l'homme), Riwâq 'Arabî, n' ${ }^{\circ}$, Le Caire, éd. du Centre du Caire pour les droits de l'homme.

AL-SAYYED SAÏD M., HASSAN B., 1995, « Hurriyyat al-sahâfa min mandhûr huqûq al-insân » (La liberté de la presse dans une perspective des droits de l'homme), Korrasat Ibn Rushd, $\mathrm{n}^{\circ} 2$, Le Caire, éd. du Centre du Caire pour les droits de l'homme.

THÉVENOT L, 1990, « L'action qui convient », Raisons pratiques, vol. 1, p. 39-69.

\section{NOTES}

1. Cela s'énonce à travers un ensemble de variations qui vont de l'altérité religieuse, en passant par l'immoralité occidentale mesurée à partir des mœurs et des conventions non explicitement religieuses, à la dénonciation de l'acculturation comme complot constant mené par les Occidentaux contre ce qui est défini comme étant le groupe d'appartenance (égyptien, arabe, ou musulman).

2. Cette rencontre inaugurale a eu lieu à Chypre, suite au refus de tous les pouvoirs arabes sans exception d'autoriser la tenue de cette réunion sur leurs territoires.

3. Par rôle majeur, $j$ 'entends la grande mobilisation appelée pour la première fois par les acteurs politiques du Machrek en général, et de l'Égypte en particulier, au nom de la défense des droits de l'homme. Par rapport à leurs homologues maghrébins, ces derniers n'adhéreront qu'assez tardivement au militantisme pour le respect des droits de l'homme.

4. Même après le lancement de l'OADH à Chypre, plusieurs obstacles politiques et légaux ont été dressés invariablement par les gouvernements arabes afin de bloquer l'institutionnalisation de cette organisation régionale.

5. C'est dans ce cadre que l'on assiste chaque année à une campagne médiatique discréditant les militants des droits de l'homme, une campagne qui atteint son apogée avec la publication du rapport annuel de l'OEDH sur l'état des droits de l'homme en Égypte.

6. Il faut dire qu'entre 1989 et 1995 aucune de ces stratégies d'action ne l'emporte sur les autres, et que chaque branche du pouvoir politique adopte, simultanément, l'une ou l'autre selon les circonstances et les enjeux. Par branche du pouvoir, j'entends le ministère de l'Intérieur, les conseillers du président pour les affaires internes ou internationales, le ministère des Affaires étrangères.

7. L'OEDH est régie par la loi des associations sociales. Une loi qui date de l'ère nassérienne et qui garantit au pouvoir politique le droit de liquider, annexer ou refuser toute association que les autorités pourraient estimer comme nuisant - ou n'apportant rien de neuf - à l'ensemble du travail associatif égyptien. L'application excessive de cette loi a mobilisé plusieurs fois l'ensemble des acteurs politiques qui ont revendiqué son abolition, ou du moins sa réforme, sans le moindre succès.

8. Le terme de professionnalisation ne doit pas s'entendre péjorativement ici. Souvent employé par les activistes égyptiens des droits de l'homme eux-mêmes, il sert à exprimer l'effort constant d'une frange importante parmi les militants pour dépasser leurs affiliations politiques et idéologiques initiales, dans le but d'éviter à leur action une déformation ou une instrumentalisation à des fins politiciennes. C'est pourquoi on le trouve souvent associé à un second terme, celui de la politisation. À ce propos, voir l'éditorial de Riwâq 'Arab, périodique publié par le centre du Caire pour les droits de l'homme, n² 2, avril 1996, p. 11 et 12.

9. Voir à ce propos la série d'articles de l'avocat Muhammad Nûr Farhat, l'un des fondateurs de l'OEDH, parus entre février et mars 1995 dans l'hebdomadaire al-Musawwar, et dans lesquels il étayait les dangers de cette ouverture du mouvement sur l'Occident, sur les trois plans politique, financier et technique. 
10. Tout un travail pourrait être réalisé sur ce point en adoptant la démarche proposée par L. Thévenot (1990, p. 39-69) au sujet des modes d'appréhension de l'action selon une perspective interactionniste (voir surtout la notion de « mise à l'épreuve du jugement »).

11. Cette description pourrait donner faussement l'impression d'une alternance entre l'idiome de la libération nationale et la dichotomie État/société civile quant aux formes entreprises d'encodage. Là, il faut préciser qu'il n'en est rien car jusqu'à aujourd'hui, l'on trouve des publications et des discours qui adhèrent à telle ou telle forme d'encodage selon le type d'engagement des militants et la nature des situations de chaque énonciation. Voir à ce sujet le livre collectif Huqûqal-insân wa ta'akhkhurMisr (Les droits de l'homme et l'arriération de l'Égypte) (1993), où l'on repère la simultanéité des deux formes d'encodage chez les auteurs des divers chapitres.

12. Dans ce livre traduit, édité, et commenté par le Centre des études et de l'information juridiques pour les droits de l'homme en 1994, le conflit Nord/Sud apparaît comme une lutte continue qu'il convient d'entretenir au nom de l'injustice régissant de plus en plus les rapports internationaux; une lutte qui, malgré les différents masques que le Nord tente de porter pour pondérer son exploitation et ses prétentions, devient manifeste dans le cadre du militantisme pour les droits de l'homme. Ce discours adressé aux militants de la gauche égyptienne tente de suggérer que seule une action active au nom du respect des droits de l'homme peut prouver l'hypocrisie occidentale et ranimer la flamme de la marche du tiers monde vers la liberté et le progrès.

13. Ces pratiques d'homologation avec le local seraient étiquetées par les activistes des droits de l'homme comme des modes de mobilisation, d'appel à l'admission de la différence au sein d'un cadre démocratique, ou, au mieux, comme tentatives de recadrage du sens.

14. Voir à ce propos Huqûq al-insân wa ta'akhkhurMisr (Les droits de l'homme et l'arriération de l'Égypte) (1993), ouvrage qui comprend une longue préface du directeur du centre édjteur et une quinzaine de contributions qui énumérant les signes de cette " arriération ».

15. Voir à ce propos le rapport publié par l'organisation égyptienne des droits de l'homme suite aux résultats des élections parlementaires de 1995 et portant le titre «La démocratie en danger : témoignage de l'OEDH ».

16. Voir la préface du livre traduit et publié par le Centre des études et de l'information juridiques, en 1994, sous le titre Khurâfât al-tanmiya aw al-sûq al-'âlamî li tijârat al-jû' (Le mythe du développement ou le marché mondial du commerce de la faim) qui met l'accent sur les droits bafoués des nations du Sud.

17. C'est effectivement le titre d'un livre écrit par trois militants des droits de l'homme, M. Tadrus, A. al-Chirbiny et A. Abdel Hakim et édité par le Centre d'études et d'information juridiques en 1995 pour débattre de la marginalisation juridique de la femme en Égypte.

18. Je tiens à souligner que l'usage de l'homologation par rapport au transnational est entendu ici d'une façon beaucoup plus circonscrite que l'acception que lui donne J.-N. Ferrie (1996). Cela dit, entreprendre un débat sur les différentes modalités de délimitation de cette opération cognitive me semble un exercice fructueux quoique dépassant le cadre de cet article.

19. Entretien avec N. al-Burâ'î, ex-secrétaire général de l'OEDH, janvier 1995.

20. Entretien avec 'Abdallah Khalîl, membre du Conseil de direction de l'OEDH, juillet 1996.

21. Voir à ce propos l'éditorial de Riwâq 'Arabî, n 2,1996.

22. Entretien avec N. al-Burâ'î, janvier 1995. 
INDEX

Mots-clés : altérité, droits de l'homme, Occident

\section{AUTEUR}

DINA EL-KHAWAGA

Cedej/Université de Helwan 